\title{
Cardiac-respiratory self-gated cine ultra-short echo time (UTE) cardiovascular magnetic resonance for assessment of functional cardiac parameters at high magnetic fields
}

Verena Hoerr ${ }^{1}$, Nina Nagelmann ${ }^{1}$, Arno Nauerth², Michael T Kuhlmann ${ }^{3}$, Jörg Stypmann ${ }^{4}$ and Cornelius Faber ${ }^{1 *}$

\begin{abstract}
Background: To overcome flow and electrocardiogram-trigger artifacts in cardiovascular magnetic resonance (CMR), we have implemented a cardiac and respiratory self-gated cine ultra-short echo time (UTE) sequence. We have assessed its performance in healthy mice by comparing the results with those obtained with a self-gated cine fast low angle shot (FLASH) sequence and with echocardiography.

Methods: 2D self-gated cine UTE (TE/TR $=314 \mu \mathrm{s} / 6.2 \mathrm{~ms}$, resolution: $129 \times 129 \mu \mathrm{m}$, scan time per slice: $5 \mathrm{~min} 5 \mathrm{sec}$ ) and self-gated cine FLASH (TE/TR $=3 \mathrm{~ms} / 6.2 \mathrm{~ms}$, resolution: $129 \times 129 \mu \mathrm{m}$, scan time per slice: 4 min $49 \mathrm{sec}$ ) images were acquired at 9.4 T. Volume of the left and right ventricular (LV, RV) myocardium as well as the end-diastolic and systolic volume was segmented manually in MR images and myocardial mass, stroke volume (SV), ejection fraction (EF) and cardiac output (CO) were determined. Statistical differences were analyzed by using Student $t$ test and Bland-Altman analyses.

Results: Self-gated cine UTE provided high quality images with high contrast-to-noise ratio (CNR) also for the RV myocardium (CNR blood-myocardium $=25.5 \pm 7.8$ ). Compared to cine FLASH, susceptibility, motion, and flow artifacts were considerably reduced due to the short TE of $314 \mu \mathrm{s}$. The aortic valve was clearly discernible over the entire cardiac cycle. Myocardial mass, SV, EF and CO determined by self-gated UTE were identical to the values measured with selfgated FLASH and showed good agreement to the results obtained by echocardiography.

Conclusions: Self-gated UTE allows for robust measurement of cardiac parameters of diagnostic interest. Image quality is superior to self-gated FLASH, rendering the method a powerful alternative for the assessment of cardiac function at high magnetic fields.
\end{abstract}

Keywords: High magnetic field, Flow artifacts, Cardiovascular magnetic resonance, Retrospective gating, Self-gated cine UTE

\section{Background}

Cardiovascular magnetic resonance (CMR) is increasingly often performed at very high magnetic field strength, up to $7 \mathrm{~T}$ for human subjects and up to $17.6 \mathrm{~T}$ for small animal models [1-7]. While higher magnetic fields hold the promise of providing better signal-to-noise ratio (SNR), higher spatial resolution or better dynamics, several

\footnotetext{
* Correspondence: faberc@uni-muenster.de

'Department of Clinical Radiology, University Hospital Muenster, Muenster, Germany

Full list of author information is available at the end of the article
}

technical issues remain. Apart from the typical high field challenges such as magnetic field inhomogeneities and specific absorption rate limitations, two major problems must be addressed for CMR in small animal models in particular: artifacts in the electrocardiogram (ECG) generated by the MR scanner itself and dynamic susceptibility artifacts derived from blood flow. To overcome problems with disturbed ECG signal retrospective gating strategies have been developed [8-10]. A very efficient approach is to use self-gating acquisition schemes, which do not require

\section{Biomed Central}


additional scans to record reference data and thus do not increase scan time. These techniques, however, do not mitigate the second crucial issue, flow artifacts. Reference data which have to be recorded during echo formation in selfgating strategies may even prolong the minimum echo time (TE) and thus lead to pronounced flow artifacts and susceptibility effects at the myocardium-lung interface. Flow artifacts may compromise exact measurement of the ventricular volumes or proper visualization of the valves. Susceptibility artifacts often impede quantification of right ventricular (RV) mass, which is already difficult due to lower contrast to other tissues at high magnetic fields.

One option to overcome these problems is to perform CMR with methods which allow for minimized TE [11]. Data acquisition with direct read out can be achieved with the technique termed ultra-short echo time (UTE) CMR. UTE sequences use center-out radial data acquisition schemes, directly recording signal after the excitation pulse [12]. Besides $M R$ of tissue with short $T_{2}$ $[13,14]$, UTE has previously been used for example for flow mapping of high velocity flow [11], MR angiography [15] or detection of iron oxide nano particles [16,17]. Although no echo is formed in UTE we will use the term TE for the delay between excitation and start of acquisition, according to established conventions.

A self-gated version of radial data acquisition has previously been proposed and shown to be able to compete in terms of SNR and contrast-to-noise ratio (CNR) with both ECG-triggered acquisition [18] and cartesian acquisition schemes [19]. Here, we have implemented and optimized a version of self-gated UTE on a $9.4 \mathrm{~T}$ small animal CMR scanner. We quantitatively and qualitatively compared the self-gated UTE sequence with a self-gated fast low angle shot (FLASH) sequence and with echocardiography in a mouse model of normal cardiac function.

\section{Methods \\ Animals}

Experiments were performed on six 12 weeks old $(22 \pm$ $1 \mathrm{~g})$ healthy female C57BL/6 wild-type mice, purchased from Harlan-Winkelmann (Borchen, Germany). All animal studies were performed with the approval of the State Review Board of North Rhine-Westphalia, Germany (Az. 87-51.04.2011.A003). Mice were imaged using self-gated cine FLASH (IntraGate, Bruker BioSpin MRI, Ettlingen, Germany) and self-gated cine UTE sequences to assess the two imaging sequences with respect to CNR and cardiac functional parameters. As comparison, mice were also measured by ultrasound.

\section{In vivo ultrasound measurements}

Cardiac Doppler-Echocardiography was performed on a specialized ultrasound biomicroscope for examination of murine models with high temporal (up to 1000 pictures per second) and spatial resolution (down to $35 \mu \mathrm{m}$ ) (VEVO 2100, VisualSonics, Toronto, Canada). Examinations were performed as previously described $[20,21]$. In brief, after anesthesia with isoflurane (2.0\% isoflurane/ $98 \% \mathrm{O}_{2}$ ) mice were shaved precordially using a commercially available electro-shaver and secured in a supine position on the heating pad of the VEVO 2100 with a feedback rectal body heat control holding a temperature of $38^{\circ} \mathrm{C}$ and controlling one-lead electrocardiogram heart rate above $400 \mathrm{bpm}$ constantly for the normal scanning time of 20 minutes. Warmed and centrifuged gel was applied to the chest of the examined mice and examinations were performed with the system's $25 \mathrm{MHz}$ linear probe. ECG-triggered parasternal short axis views of the whole mouse heart were obtained in steps of $100 \mu \mathrm{m}$ together with a parasternal long axis view. Routinely pulsed wave Doppler signals of the velocity time integral of the mitral valve inflow, the aortic outflow and the pulmonic outflow were obtained.

\section{Ultrasound Image analysis}

Ultrasound image analysis was performed independently by two experienced ultrasound readers using the VEVO 2100 implemented image analysis. 2D images were manually segmented for end-diastolic and end-systolic heart cycle. Velocity time integrals of the valvular blood flow signals were manually segmented, too. Calculations of the volumes followed Teichholz's formula (m-mode) and Simpson's formula (2D). Calculations of cardiac output (CO), stroke volume (SV) were based on either the 2D measurements or the continuous flow formula.

\section{In vivo $\mathrm{CMR}$ measurements}

CMR images of healthy C57BL/6 mice were acquired at 9.4 $\mathrm{T}$ on a Bruker BioSpec 94/20 (Ettlingen, Germany) equipped with a $1 \mathrm{~T} / \mathrm{m}$ gradient system and a $35 \mathrm{~mm}$ volume coil. The MR system was interfaced to a console running ParaVision software 5.1 including the IntraGate software for sequence acquisition and reconstruction. For MR acquisition a cardiac and respiratory self-gated UTE sequence was implemented and animals were imaged with the following parameters: slice thickness, $1 \mathrm{~mm}$; number of slices, 9; matrix size, $156 \times 156$; field of view, $20 \times$ $20 \mathrm{~mm}^{2}$; averages, 1; TE, $0.314 \mathrm{~ms}$; TR, $6.2 \mathrm{~ms}$; flip angle, $15^{\circ}$; pulse shape, gaussian; pulse length, $0.3 \mathrm{~ms}$; number of projections, 246; polar undersampling, 2.0; number of movie cycles, 200; acquisition bandwidth, $100 \mathrm{kHz}$; scan time per slice, $5 \mathrm{~min} 5 \mathrm{sec}$. No half-pulse was used. Gradients were ramped in a $160-\mu$ s delay, which allowed for gradient rising to full amplitude (115 $\mu \mathrm{s}, 128 \mu \mathrm{s}, 132 \mu \mathrm{s}$ for the three axes) and preemphasis corrections. Kspace was sampled with constant increments of the backprojection angle. After the acquisition a delay of $2.5 \mathrm{~ms}$ was applied to achieve the same TR as in the 
FLASH sequence. For reconstruction, interpolation of the radially sampled k-space data onto a Cartesian grid was performed by a Kaiser Bessel Kernel regridding algorithm [22-24]. A stack of contiguous short-axis slices was acquired to cover the entire right and left ventricles. Image quality as well as functional and morphological cardiac parameters were assessed and compared to those obtained by using the self-gated cine FLASH (IntraGate FLASH) sequence with the following parameters: slice thickness, $1 \mathrm{~mm}$; number of slices, 9; matrix size, $232 \times$ 232; field of view, $30 \times 30 \mathrm{~mm}^{2}$; averages, 1 ; TE, $3 \mathrm{~ms}$; flip angle, $15^{\circ}$; pulse shape, gaussian; pulse length, $0.5 \mathrm{~ms}$; TR, $6.2 \mathrm{~ms}$; number of repetitions, 200; acquisition bandwidth, $75 \mathrm{kHz}$; scan time per slice, $4 \mathrm{~min} 49 \mathrm{sec}$.

During CMR measurements, animals were anesthetized with $2 \%$ isoflurane and were monitored for core body temperature and respiration rate using a CMR compatible monitoring system (SA Instruments, Stony Brook, NY).

\section{CMR techniques and reconstruction}

For retrospective sorting of the acquired image data, self-gated CMR sequences rely on additional data that are recorded at some point during the repetition time. In the IntraGate FLASH sequence these data are recorded either during refocusing of the slice signal or by an additional navigator slice. If the navigator signal is derived from the slice signal, the phase encoding and pre-read dephase gradients need to be separated from the slice rephase gradient, which leads to a prolonged TE (Figure 1A). The excitation and recording of the additional navigator slice signal leads to longer minimum repetition time TR (Figure 1B).

In self-gated cine UTE sequences, however, the navigator signal is derived directly from the MR signal of the image acquisition. During center-out radial k-space sampling each spoke contains the central k-space in its origin, which leads to a further shortening of TE (Figure 1C).

Since the navigator signals have a constant encoding, they record all disturbances that occur during the measurement. Presuming that respiratory motion, cardiac motion and/or pulsatile blood flow occurs within the slice that is used to derive the navigator, a respiration and cardiac signal can be deduced and used for the retrospective reconstruction of a cardiac cine.

\section{CMR analysis}

CNR as measure for image quality was calculated between the left and right myocardial wall and the ventricles for the end-diastolic and end-systolic frame as $\mathrm{CNR}=$ (signal intensity_blood - signal intensity_myocardium)/standard deviation_noise. Signal intensities and standard deviations were determined using the Amira software (Version 5.4.0,
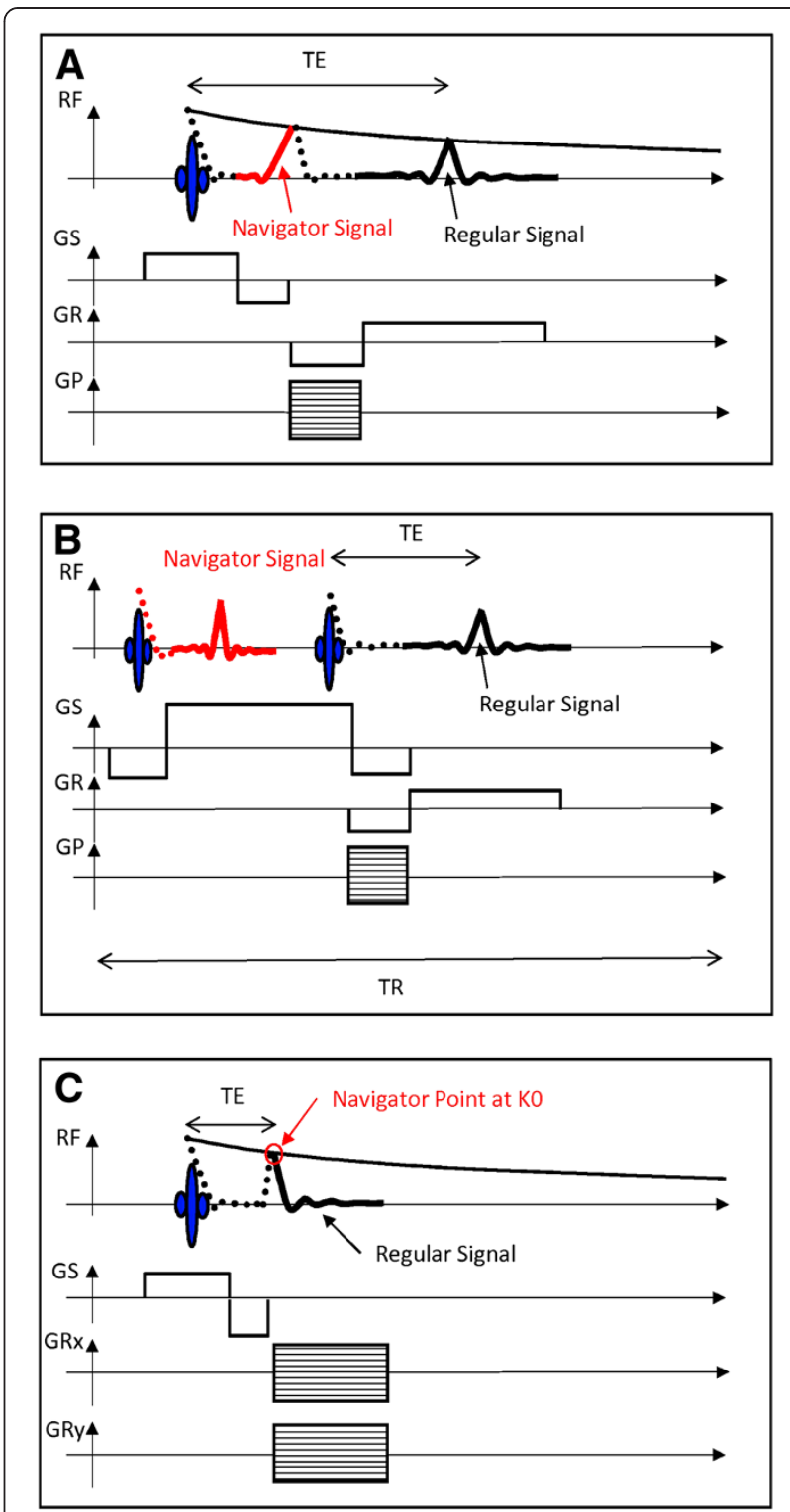

Figure 1 Illustration of the recorded navigator in self-gated cine FLASH and UTE sequences. In the self-gated cine FLASH sequence $(\mathbf{A}, \mathbf{B})$ the navigator is either derived from the refocusing slice signal (A), which increases minimum $\mathrm{TE}$, or taken from a navigator slice (B), which increases minimum TR. In the self-gated UTE sequence $(\mathbf{C})$ the first data point of each acquisition contains all motion information and is used as navigator point, without requiring additional experimental time.

Visage Imaging $\mathrm{GmbH}$, Berlin, Germany) as mean value over all segmented slices.

In UTE images CNR was also determined between the right myocardial wall and the adjacent tissues of lung, liver and muscle. Volumetric analysis of the left and right myocardium, and ventricles was performed using the Amira software. The regions of interest in the MR 
images were selected manually on the end-diastole and end-systole frame of each slice by tracing the epicardial and endocardial borders. In order to obtain the global parameters of the entire heart the volume for each frame was calculated as the sum of the area of interest in each slice multiplied by the slice thickness. SV, CO per minute and the ejection faction (EF) were calculated from the blood volume, determined in the end-systolic and end-diastolic phase.

Left ventricular (LV) and RV mass was calculated by multiplying the volume of the LV and RV myocardium by the tissue density of $1.05 \mathrm{~g} / \mathrm{cm}^{3}$.

In addition to the determination of the functional cardiac parameters, the self-gated cine FLASH and UTE sequence was assessed with respect to flow artifacts (Q1), their boundary between the ventricular blood and the myocardium including the papillary muscles $(\mathrm{Q} 2)$, susceptibility artifacts at the myocardium-lung interface (Q3), their interference with the depiction of the RV myocardium (Q4), acquisition artifacts (Q5) and the overall suitability for segmentation of the LV cavity and the myocardium using Segment (Version 1.9, Medviso $A B$, Lund, Sweden) and Amira software (Q6). The rating was performed in a blinded fashion using a 5-level scale from one to five $(1=$ best, $5=$ worst $)$. Statistical differences between both the functional and the quantitative values obtained for the two sequences were analyzed using Student $t$ test. All aspects of analysis were performed by two CMR specialists independently.

\section{Results}

Image quality

Image contrast

Stacks of high resolution short-axis cardiac images covering the entire mouse heart were acquired from six healthy mice using the $2 \mathrm{D}$ cardiac-respiratory self-gated cine UTE sequence. Image quality was assessed with respect to $\mathrm{CNR}$, and was compared with the results obtained with a conventional cartesian gradient echo (FLASH) sequence that is routinely used in CMR on mouse models. Both sequences acquired the same number of frames over the cardiac cycle with the same spatial resolution $\left((129 \mu \mathrm{m})^{2}\right.$ in-plane; $1 \mathrm{~mm}$ slice $)$ in the same acquisition time of 5 min per slice. Both sequences yielded high quality images, with CNR values above 20 (Table 1). A lower noise level was observed in the gradient echo cine images, resulting in higher CNR values throughout. However, for the UTE images excellent contrast was observed between the right myocardium and the surrounding tissue (Table 2).

\section{Artifacts}

Figure 2A shows four different frames of cine UTE and FLASH images during the cardiac cycle, exemplified in
Table 1 CNR values between the blood in the ventricles and the myocardial wall

\begin{tabular}{lll}
\hline CNR $^{\S}$ & End-diastole $^{\mathbf{5}}$ & End-systole $^{\mathbf{5}}$ \\
\hline UTE (left ventricle - left myocardium) & $22.7 \pm 5.3$ & $21.1 \pm 4.5$ \\
UTE (right ventricle - right myocardium) & $25.5 \pm 7.8$ & $21.5 \pm 5.2$ \\
FLASH (left ventricle - left myocardium) & $40.8 \pm 12.0$ & $36.7 \pm 11.0$ \\
FLASH (right ventricle - right & $39.6 \pm 11.0$ & $33.1 \pm 9.7$ \\
myocardium) & &
\end{tabular}

${ }_{5}^{5}$ Contrast-to-noise ratio (CNR) was measured in the end-diastolic and endsystolic phase by self-gated FLASH and self-gated UTE sequences.

\$Values are given as mean \pm standard deviation values, averaged over six healthy C57BL/6 mice.

one central short-axis slice. To assess the image quality of the two sequences at different cardiac phases two independent readers rated images at the end-diastolic, ejection, end-systolic and the inflow phase with respect to six quality criteria. Results of the qualitative assessments in the end-diastolic and inflow phase are summarized in Figure 2B. Similar to previous studies $[8,25]$ flow artifacts were in particular observed in the gradient echo images during the inflow phase $(\mathrm{Q} 1$ : reader 1 [R1]: $\mathrm{p}=0.000018$, reader $2[\mathrm{R} 2]: \mathrm{p}=0.000007)$. Further, pronounced susceptibility artifacts were observed in the $\mathrm{T}_{2}{ }^{*}$ weighted acquisitions in all cardiac phases, in particular at tissue interfaces which affected mostly the right myocardium in proximity to the lung (end-diastole: Q3: R1:p= 0.022, R2:p $=0.0079$; ejection: Q3: R1:p $=0.0056, \mathrm{R} 2: \mathrm{p}=$ 0.000046; end-systole: $\mathrm{Q} 3: \mathrm{R} 1 \mathrm{p}=0.0012, \mathrm{R} 2 \mathrm{p}=0.0046$; inflow: Q3: $\mathrm{R} 1: \mathrm{p}=0.0014, \mathrm{R} 2 \mathrm{p}=0.000035$ ). The importance of minimized TE is illustrated in Figure 2C. Longer $\mathrm{TE}$, as were required in sequences with echo formation, yielded images with substantially stronger flow artifacts.

\section{Functional and morphological characterization Cardiac function}

LV and RV heart function was assessed on the basis of the cardiac images acquired with either the $\mathrm{T}_{2}{ }^{*}$-weighted FLASH or the UTE sequence. LV and RV myocardium as well as cavity were manually segmented and myocardial mass, SV, EF and CO were calculated. The LV

Table 2 CNR values between the right ventricular (RV) myocardium and surrounding tissues

\begin{tabular}{lll}
\hline CNR $^{\S}$ & End-diastole $^{\text {\$ }}$ & End-systole $^{\mathbf{5}}$ \\
\hline RV myocardium - muscle & $14.9 \pm 3.1$ & $14.9 \pm 4.9$ \\
RV myocardium - liver & $7.0 \pm 2.2$ & $6.7 \pm 2.7$ \\
RV myocardium - lung & $7.5 \pm 3.2$ & $7.5 \pm 3.3$ \\
\hline
\end{tabular}

${ }^{5}$ Contrast-to-noise ratio (CNR) was measured in self-gated UTE images.

${ }^{\$}$ Values are given as mean \pm standard deviation values averaged over six healthy C57BL/6 mice. 

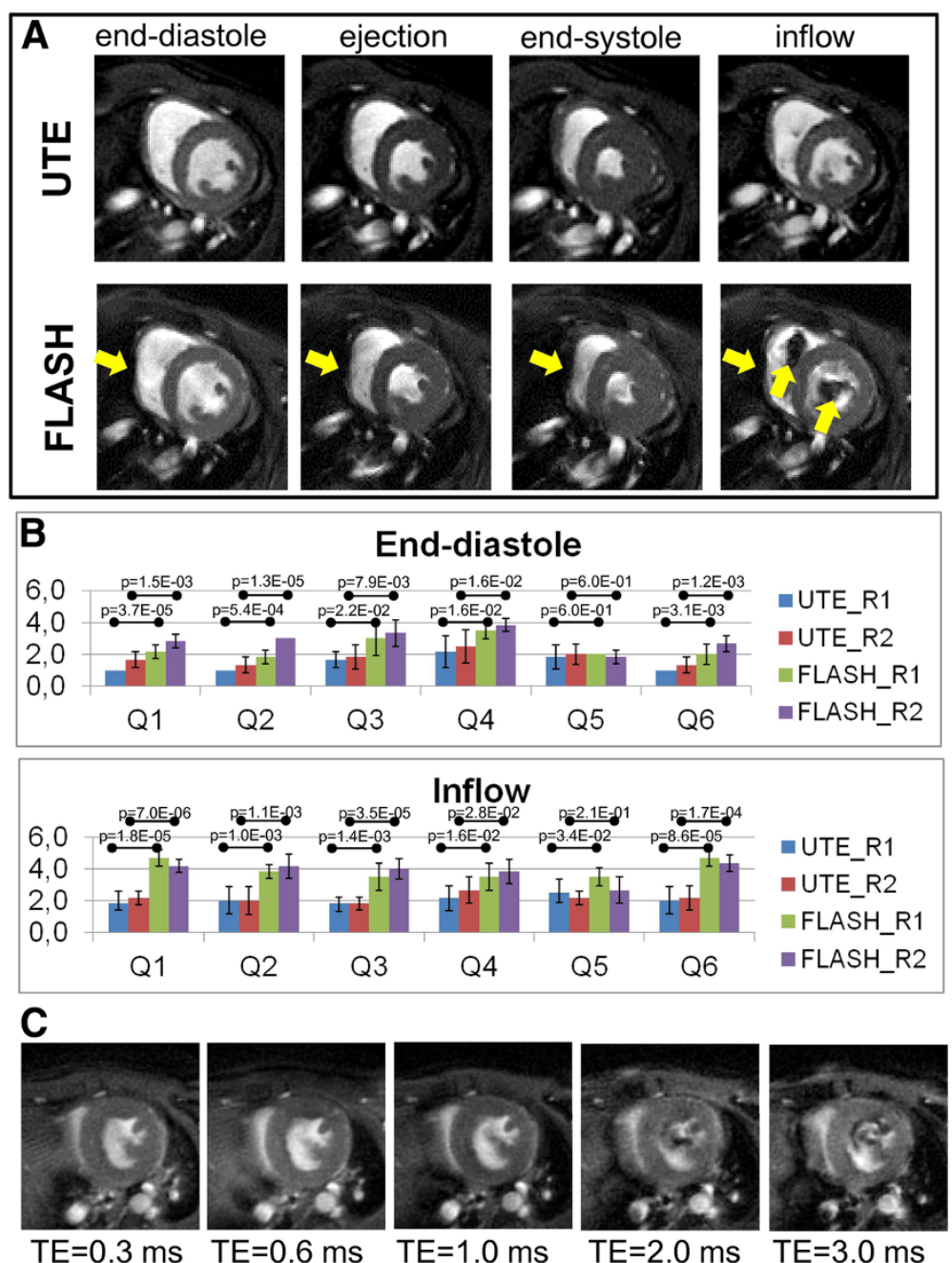

Figure 2 Assessment of mid-ventricular short-axis views of a mouse heart in different cardiac phases. (A) Exemplary self-gated cine FLASH and UTE images in the end-diastolic, ejection, end-systolic and inflow phase of a cardiac cycle. Flow and susceptibility artifacts were more pronounced in self-gated FLASH images than in UTE images and are highlighted by yellow arrows. (B) Results of the qualitative assessment of both self-gated UTE and FLASH images in the end-diastolic and inflow phase, performed by two independent readers (R1 and R2) using a 5-level scale ( 1 = best, 5 = worst). The image quality was assessed with respect to flow artifacts (Q1), their boundary between the ventricular blood and the myocardium including the papillary muscles (Q2), susceptibility artifacts at the myocardium-lung interface (Q3), their interference with the depiction of the RV myocardium (Q4), acquisition artifacts (Q5) and the overall suitability for segmentation of the LV cavity and the myocardium using Segment and Amira software (Q6). Results were averaged over images of six healthy C57BL/6 mice. (C) Self-gated UTE images in the cardiac inflow phase acquired with echo times of $0.314 \mathrm{~ms}, 0.6 \mathrm{~ms}, 1 \mathrm{~ms}, 2 \mathrm{~ms}, 3 \mathrm{~ms}$.

morphological and functional parameters measured with self-gated UTE perfectly reproduced the values measured with the self-gated gradient echo method. For the RV parameters slightly but not significantly increased values were observed for myocardial mass $(\mathrm{p}=0.10)$ and EF $(\mathrm{p}=0.34)$ (Table 3). Bland-Altman analysis for left and right ventricular mass, SV, $\mathrm{CO}$, and $\mathrm{EF}$ suggested high reproducibility and negligible bias of the data (not shown). LV and RV cardiac function was also investigated by $2 \mathrm{D}$ echocardiography as the clinical reference. Cardiac parameters obtained by echocardiography and CMR showed excellent agreement for the myocardial mass, SV and CO, but differed significantly for the EF (echocardiography vs. FLASH, $\mathrm{p}=0.000022$ ).

\section{Morphology}

As shown in Figure 2A images acquired with self-gated UTE were virtually free from artifacts and provided high tissue contrast, which allowed for detailed visualization of anatomical structures of the aortic valves (Figure 3A). Left, right and posterior semilunar cusps were clearly separated by the aortic-mitral septum. While flow artifacts in conventional $T_{2}$ and $T_{2}{ }^{*}$ weighted images hampered the visualization of the aortic valves in open position 
Table 3 Results of left and right ventricular (LV, RV) functional analysis performed by the two CMR sequences, self-gated FLASH and self-gated UTE as well as by echocardiography

$$
\text { Echocardio-graphy }^{\S} \text { CMR Self-gated cine FLASH }{ }^{\S} \text { CMR Self-gated cine UTE }{ }^{\S} \text { p-values FLASH-UTE/FLASH- }
$$
Ultrasound $^{\$}$

\section{LV morphology and function}

Mass [mg]

$91 \pm 11$

Stroke volume (SV) $[\mu l]$

$35.0 \pm 5.4$

Ejection fraction (EF) [\%]

$54.7 \pm 4.8$

Cardiac output (CO) $[\mathrm{ml} / \mathrm{min}] \quad 16.6 \pm 3.2$

RV morphology and function

$\begin{array}{lll}\text { Mass }[\mathrm{mg}] & - & 15.0 \pm 4 \\ \text { Stroke volume }(\mathrm{SV})[\mu \mathrm{l}] & 31.7 \pm 7.5 \text { (Doppler) } & 22.9 \pm 3.5 \\ \text { Ejection fraction (EF) }[\%] & - & 72.4 \pm 6.1 \\ \text { Cardiac output (CO) }[\mathrm{ml} / \mathrm{min}] & 13.2 \pm 3.2 \text { (Doppler) } & 11.0 \pm 2.2\end{array}$

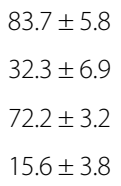

$\begin{array}{ll}83.6 \pm 3.0 & 0.98 / 0.20 \\ 32.3 \pm 6.5 & 0.99 / 0.47 \\ 71.8 \pm 2.2 & 0.78 / 0.000022 \\ 15.6 \pm 3.8 & 0.99 / 0.63\end{array}$

$19.3 \pm 3.7$

0.10

$22.6 \pm 3.9$

$0.89 / 0.026$

$69.6 \pm 3.2$

0.34

$10.9 \pm 2.1$

$0.89 / 0.20$

$\sqrt{5}$ Values are given as mean \pm standard deviation values averaged over six healthy C57BL/6 mice.

(Figure 3C), UTE images allowed for visualization of the cardiac valves over the entire cardiac cycle (Fig ure 3B).

\section{Discussion}

We have implemented and used a self-gated UTE sequence for assessment of functional cardiac parameters in mice at a magnetic field strength of 9.4 T. The center-out acquisition scheme allowed for a TE of only $314 \mu$ s, which minimized typical high field problems like flow and susceptibility artifacts. A further reduction in TE can be achieved when a half-pulse excitation is used [26,27].
However, since two scans have to be added pairwise, even small displacements from cardiac motion may lead to additional artifacts. Furthermore, half-pulse excitation doubles total scan time. We found that already without half-pulse excitation, a TE of $314 \mu$ s provided sufficient image quality, TE could be reduced substantially compared to selfgated cartesian sampling schemes, because no time for echo formation and acquisition of navigator data was required. Also compared to a previous implementation of self-gated radial acquisition, TE was reduced by a factor of four $[18,19]$, and also radial FLASH acquisitions [28-30]
A

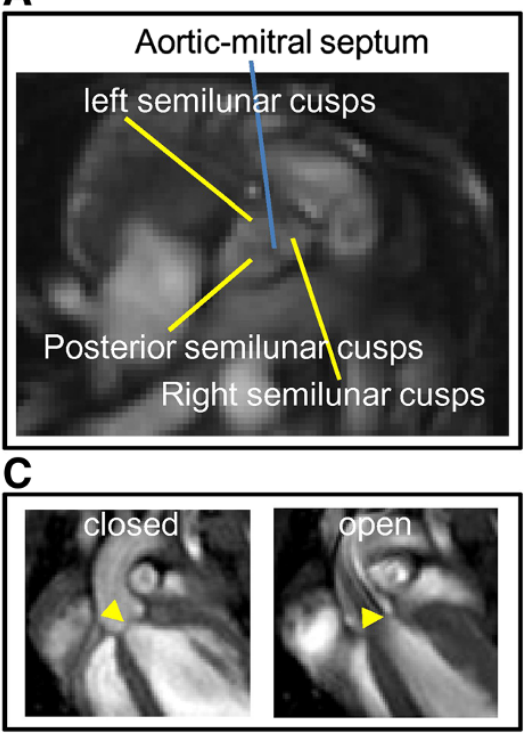

B

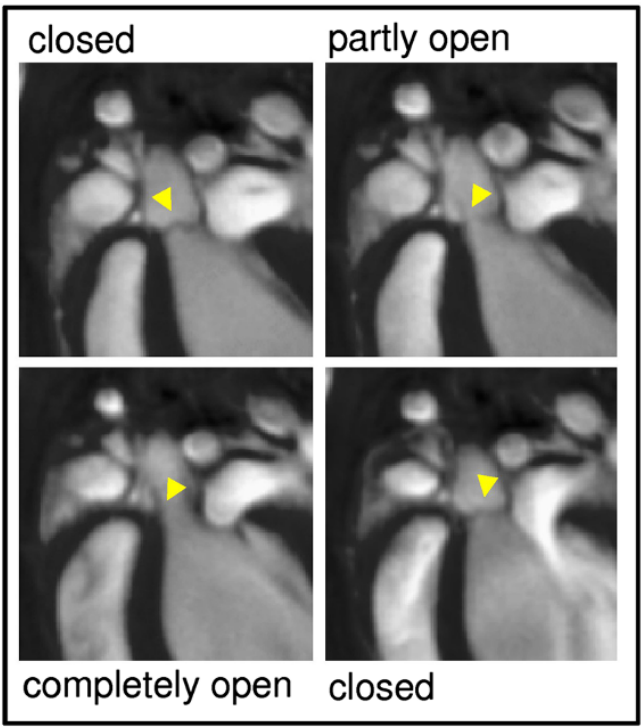

Figure 3 Self-gated cine FLASH and UTE images of the aortic valve. Self-gated UTE images (A) in short-axis orientation visualizing the aorticmitral septum and (B) long-axis view of the aortic valve (yellow arrowhead) in different states during the heart cycle. (C) Self-gated FLASH images of the aortic valve (yellow arrowhead) in closed and open position. 
will not be able to reach TE values as short as reported here. Due to the increased artifacts at longer TE (see Figure 2C) we did not compare our method with radial FLASH, although a reduction in scan time would have been possible.

As expected, the minimized TE almost completely suppressed flow and susceptibility artifacts (Figure 2) and thus confirmed the robustness of UTE methods in the presence of flow [11]. Although excellent image quality can be obtained by cartesian sampling schemes at even higher magnetic fields [31-38], we found that identification of small morphological structures like valves or papillary muscles was improved by using the self-gated UTE sequence. With UTE small structures were visualized in great detail, although the SNR was reduced by a factor of two, compared to self-gated FLASH. In particular for the application of automated segmentation algorithms, the improved image quality may outbalance the lower SNR [39]. The reduced SNR in the UTE images stems from two origins, which each is responsible for roughly a factor square-root of two reduction. To achieve the same scan time with UTE, polar undersampling by a factor of two was chosen, and further, FLASH data could be acquired with a slightly lower bandwidth (75 kHz vs. $100 \mathrm{kHz}$ ).

For assessment of cardiac functional indices it has previously been found that self-gating sequences yield comparable values to those obtained with prospective triggering [9]. Our data confirm that indices obtained with both self-gated UTE and self-gated FLASH agree perfectly with data from echocardiography (Table 3 ). The systematic overestimation of the EF by CMR has been observed in accordance with previous reports [40]. One great advantage of self-gated data acquisition and any other restrospective reconstruction in general is that temporal resolution (i. e. number of cardiac frames) has not to be fixed at the beginning of the measurement $[41,42]$. As long as sufficient repetitions have been recorded the number of reconstructed frames is only limited by the resulting SNR. As recently shown, temporal resolutions of nearly $1 \mathrm{~ms}$ can be achieved, which allows for assessment of diastolic dysfunction [43], a condition that requires elaborate experimental design if studied with prospectively triggered CMR [40].

A recent study has found that self-gated sequences are more robust and more efficient for imaging of the aorta in mice [25]. However, prospective methods have higher demands on the ability, experience and diligence of the experimenter. Slightly imperfect placement of the electrodes or adjustment of scan parameters may have great impact on image quality. Self-gating methods, on the opposite, have proven to be very robust. While for small animal CMR this issue is less critical, it might be for ultrahigh field CMR on human subjects. There, self-gating avoids problems in ECG-signal analysis that have previously been found to be critical [44], and do not require the use of alternative gating methods. Besides the convenient and efficient use for accurate characterization of mouse models of cardiac disease, we therefore see potential for self-gated UTE in clinical application at ultrahigh field.

\section{Conclusion}

In conclusion, we have shown that self-gated UTE is a reliable and accurate technique in CMR and allows for robust and versatile measurement of most cardiac parameters of diagnostic interest. The sequence provided high quality images and compared to self-gated FLASH, susceptibility and flow artifacts were significantly decreased in particular during the inflow phase of the cardiac cycle due to the use of short TE. Enhanced image quality allowed for reliable quantification of both the left and right ventricular function and made the visualization of small anatomical structures such as the papillary muscles and the cardiac valve possible during one cardiac cycle.

\section{Competing interests}

The authors VH, NN, MK, JS and CF declare that they have no competing interests. AN is an employee of Bruker BioSpin MRI GmbH.

\section{Authors' contributions}

$\mathrm{VH}$ coordinated the study, designed experiments, performed CMR, collected and analyzed data and wrote the manuscript, NN designed experiments, performed CMR and analyzed data, AN wrote and implemented the selfgated UTE pulse sequence program, MK designed experiments, JS performed echocardiography and analyzed data, CF designed experiments and wrote the manuscript. All authors edited the manuscript. All authors read and approved the final manuscript.

\section{Acknowledgement}

This work was supported by the German research Foundation (SFB 656, Z2 and C3 projects) and the interdisciplinary center for clinical research (IZKF) Münster (PIX, core unit SAMRI and ECHO). The authors thank Richard Holtmeier and Justine Ongey for technical support.

\section{Author details}

${ }^{1}$ Department of Clinical Radiology, University Hospital Muenster, Muenster, Germany. ${ }^{2}$ Bruker BioSpin MRI GmbH, Ettlingen, Germany. 'European Institute for Molecular Imaging, Muenster, Germany. ${ }^{4}$ Department of Cardiovascular Medicine, Division of Cardiology, University Hospital Muenster, Muenster, Germany.

Received: 14 May 2013 Accepted: 19 June 2013

Published: 4 July 2013

\section{References}

1. Niendorf T, Sodickson DK, Krombach GA, Schulz-Menger J. Toward cardiovascular MRI at $7 \mathrm{~T}$ : clinical needs, technical solutions and research promises. Eur Radiol. 2010; 20:2806-16.

2. Moser E, Stahlberg F, Ladd ME, Trattnig S. 7-T MR-from research to clinical applications? NMR Biomed. 2012; 25:695-716.

3. Bun SS, Kober F, Jacquier A, Espinosa L, Kalifa J, Bonzi MF, Kopp F, Lalevee N, Zaffran S, Deharo JC, Cozzone PJ, Bernard M. Value of in vivo T2 measurement for myocardial fibrosis assessment in diabetic mice at 11.75 T. Invest Radiol. 2012; 47:319-23.

4. Bohl S, Lygate CA, Barnes H, Medway D, Stork LA, Schulz-Menger J, Neubauer S, Schneider JE. Advanced methods for quantification of infarct size in mice using three-dimensional high-field late gadolinium enhancement MRI. Am J Physiol Heart Circ Physiol. 2009; 296:H1200-08. 
5. Hankiewicz JH, Lewandowski ED. Improved cardiac tagging resolution at ultra-high magnetic field elucidates transmural differences in principal strain in the mouse heart and reduced stretch in dilated cardiomyopathy. J Cardiovasc Magn Reson. 2007; 9:883-90.

6. Neuberger T, Greiser A, Nahrendorf M, Jakob PM, Faber C, Webb AG. 23Na microscopy of the mouse heart in vivo using density-weighted chemical shift imaging. Magn Reson Mater Phys Biol Med. 2004; 17:196-200.

7. Aguor EN, Arslan F, van de Kolk CW, Nederhoff MG, Doevendans PA, van Echteld CJ, Pasterkamp G, Strijkers GJ. Quantitative T 2* assessment of acute and chronic myocardial ischemia/reperfusion injury in mice. Magn Reson Mater Phys Biol Med. 2012; 25:369-79.

8. Bovens SM, te Boekhorst BC, den Ouden K, van de Kolk KW, Nauerth A, Nederhoff MG, Pasterkamp G, ten Hove M, van Echteld CJ. Evaluation of infarcted murine heart function: comparison of prospectively triggered with self-gated MRI. NMR Biomed. 2011; 24:307-15.

9. Heijman E, de Graaf W, Niessen P, Nauerth A, van Eys G, de Graaf L, Nicolay K, Strijkers GJ. Comparison between prospective and retrospective triggering for mouse cardiac MRI. NMR Biomed. 2007; 20:439-47.

10. Lai P, Larson AC, Park J, Carr JC, Li D. Respiratory self-gated fourdimensional coronary MR angiography: a feasibility study. Magn Reson Med. 2008; 59:1378-85.

11. O'Brien KR, Gabriel RS, Greiser A, Cowan BR, Young AA, Kerr AJ. Aortic valve stenotic area calculation from phase contrast cardiovascular magnetic resonance: the importance of short echo time. J Cardiovasc Magn Reson. 2009; 11:49-60.

12. Robson MD, Gatehouse PD, Bydder M, Bydder GM. Magnetic resonance: An introduction to ultrashort TE (UTE) imaging. J Comput Assist Tomogr. 2003; 27:825-46.

13. Rahmer J, Börnert $P$, Dries SP. Assessment of anterior cruciate ligament reconstruction using 3D ultrashort echo-time MR imaging. J Magn Reson Imaging. 2009; 29:443-48.

14. Takahashi M, Togao O, Obara M, van Cauteren M, Ohno Y, Doi S, Kuro-o M, Malloy C, Hsia CC, Dimitrov I. Ultra-short echo time (UTE) MR imaging of the lung: comparison between normal and emphysematous lungs in mutant mice. J Magn Reson Imaging. 2010; 32:326-33.

15. Nielsen HT, Gold GE, Olcott EW, Pauly JM, Nishimura DG. Ultrashort echotime 2D time-of-flight MR angiography using a half-pulse excitation. Magn Reson Med. 1999; 41:591-99.

16. Girard OM, Du J, Agemy L, Sugahara KN, Kotamraju VR, Ruoslahti E, Bydder GM, Mattrey RF. Optimization of iron oxide nanoparticle detection using ultrashort echo time pulse sequences: comparison of $\mathrm{T}(1), \mathrm{T}(2)^{*}$, and synergistic $\mathrm{T}(1)$ - $\mathrm{T}$ (2)* contrast mechanisms. Magn Reson Med. 2011; 65:1649-60.

17. Strobel K, Hoerr V, Schmid F, Wachsmuth L, Löffler B, Faber C. Early detection of lung inflammation: Exploiting $T(1)$-effects of iron oxide particles using UTE MRI. Magn Reson Med. 2012; 68:1924-31.

18. Hiba B, Richard N, Janier M, Croisille P. Cardiac and respiratory double selfgated cine MRI in the mouse at 7 T. Magn Reson Med. 2006; 55:506-13.

19. Hiba B, Richard N, Thibault H, Janier M. Cardiac and respiratory self-gated cine MRI in the mouse: comparison between radial and rectilinear techniques at 7T. Magn Reson Med. 2007; 58:745-53.

20. Stypmann J. Doppler ultrasound in mice. Echocardiography. 2007; 24:97-112.

21. Stypmann J, Engelen MA, Troatz C, Rothenburger M, Eckardt L, Tiemann K. Echocardiographic assessment of global left ventricular function in mice. Lab Anim. 2009; 43:127-37.

22. O'Sullivan JD. A fast sinc function gridding algorithm for fourier inversion in computer tomography. IEEE Trans Med Imaging. 1985; 4:200-07.

23. Jackson II, Meyer CH, Nishimura DG, Macovski A. Selection of a convolution function for Fourier inversion using gridding [computerised tomography application]. IEEE Trans Med Imaging. 1991; 10:473-78.

24. Beatty PJ, Nishimura DG, Pauly JM. Rapid gridding reconstruction with a minimal oversampling ratio. IEEE Trans Med Imaging. 2005; 24:799-808.

25. Fries P, Massmann A, Seidel R, Müller A, Stroeder J, Custodis F, Reil J, Schneider G, Buecker A. Comparison of retrospectively self-gated and prospectively triggered FLASH sequences for cine imaging of the aorta in mice at 9.4 Tesla. Invest Radiol. 2012; 47:259-66.

26. Du J, Bydder GM. Qualitative and quantitative ultrashort-TE MRI of cortical bone. NMR Biomed. 2013; 26:489-506.

27. Robson MD, Gatehouse PD. Consequences of $\mathrm{T} 2$ relaxation during halfpulse slice selection for ultrashort TE imaging. Magn Reson Med. 2010; 64:610-15.
28. Uecker M, Zhang S, Voit D, Karaus A, Merboldt KD, Frahm J. Real-time MRI at a resolution of 20 ms. NMR Biomed. 2010; 23:986-94.

29. Niebergall A, Zhang S, Kunay E, Keydana G, Job M, Uecker M, Frahm J. Realtime MRI of speaking at a resolution of 33 ms: undersampled radial FLASH with nonlinear inverse reconstruction. Magn Reson Med. 2013; 69:477-85.

30. Vakil P, Ansari SA, Hurley MC, Bhat H, Batjer HH, Bendok BR, Eddleman CS, Carroll TJ. Magnetization spoiling in radial FLASH contrast-enhanced MR digital subtraction angiography. J Magn Reson Imaging. 2012; 36:249-58.

31. Schneider JE, Cassidy PJ, Lygate C, Tyler DJ, Wiesmann F, Grieve SM, Hulbert K, Clarke K, Neubauer S. Fast, high-resolution in vivo cine magnetic resonance imaging in normal and failing mouse hearts on a vertical 11.7 T system. J Magn Reson Imaging. 2003; 18:691-701.

32. Cassidy PJ, Schneider JE, Grieve SM, Lygate C, Neubauer S, Clarke K. Assessment of motion gating strategies for mouse magnetic resonance at high magnetic fields. J Magn Reson Imaging. 2004; 19:229-37.

33. Tyler DJ, Lygate CA, Schneider JE, Cassidy PJ, Neubauer S, Clarke K. CINE-MR imaging of the normal and infarcted rat heart using an $11.7 \mathrm{~T}$ vertical bore MR system. J Cardiovasc Magn Reson. 2006; 8:327-33.

34. Herold V, Mörchel P, Faber C, Rommel E, Haase A, Jakob PM. In vivo quantitative three-dimensional motion mapping of the murine myocardium with PC-MRI at 17.6 T. Magn Reson Med. 2006; 55:1058-64.

35. Herold V, Wellen J, Ziener CH, Weber T, Hiller KH, Nordbeck P, Rommel E, Haase A, Bauer WR, Jakob PM, Sarkar SK. In vivo comparison of atherosclerotic plaque progression with vessel wall strain and blood flow velocity in apoE(-/-) mice with MR microscopy at $17.6 \mathrm{~T}$. Magn Reson Mater Phys Biol Med. 2009; 22:159-66.

36. Herold V, Parczyk M, Mörchel P, Ziener CH, Klug G, Bauer WR, Rommel E, Jakob PM. In vivo measurement of local aortic pulse-wave velocity in mice with MR microscopy at 17.6 Tesla. Magn Reson Med. 2009; 61:1293-99.

37. Parczyk M, Herold V, Klug G, Bauer WR, Rommel E, Jakob PM. Regional in vivo transit time measurements of aortic pulse wave velocity in mice with highfield CMR at 17.6 Tesla. J Cardiovasc Magn Reson. 2010; 12:72-80.

38. Schneider JE, Hulbert KJ, Lygate CA, Ten Hove M, Cassidy PJ, Clarke K, Neubauer S. Long-term stability of cardiac function in normal and chronically failing mouse hearts in a vertical-bore MR system. Magn Reson Mater Phys Biol Med. 2004; 17:162-69.

39. Heijman E, Aben JP, Penners C, Niessen P, Guillaume R, van Eys G, Nicolay K, Strijkers GJ. Evaluation of manual and automatic segmentation of the mouse heart from CINE MR images. J Magn Reson Imaging. 2008; 27:86-93.

40. Stuckey DJ, Carr CA, Tyler DJ, Clarke K. Cine-MRI versus two-dimensional echocardiography to measure in vivo left ventricular function in rat heart. NMR Biomed. 2008; 21:765-72.

41. Bucholz E, Ghaghada K, Qi Y, Mukundan S, Johnson GA. Four-dimensional MR microscopy of the mouse heart using radial acquisition and liposomal gadolinium contrast agent. Magn Reson Med. 2008; 60:111-18.

42. Bucholz E, Ghaghada K, Qi Y, Mukundan S, Rockman HA, Johnson GA. Cardiovascular phenotyping of the mouse heart using a 4D radial acquisition and liposomal Gd-DTPA-BMA. Magn Reson Med. 2010; 63:979-87.

43. Coolen BF, Abdurrachim D, Motaal AG, Nicolay K, Prompers JJ, Strijkers GJ. High frame rate retrospectively triggered Cine MRI for assessment of murine diastolic function. Magn Reson Med. 2013; 69:648-56.

44. Suttie JJ, Delabarre L, Pitcher A, van de Moortele PF, Dass S, Snyder CJ, Francis JM, Metzger GJ, Weale P, Ugurbil K, Neubauer S, Robson M, Vaughan T. 7 Tesla (T) human cardiovascular magnetic resonance imaging using FLASH and SSFP to assess cardiac function: validation against $1.5 \mathrm{~T}$ and 3 T. NMR Biomed. 2012; 25:27-34.

doi:10.1186/1532-429X-15-59

Cite this article as: Hoerr et al.: Cardiac-respiratory self-gated cine ultrashort echo time (UTE) cardiovascular magnetic resonance for assessment of functional cardiac parameters at high magnetic fields. Journal of Cardiovascular Magnetic Resonance 2013 15:59. 